\title{
LINEAR QUADRATIC OPTIMIZATION FOR FRACTIONAL ORDER DIFFERENTIAL ALGEBRAIC SYSTEM OF RIEMANN- LIOUVILLE TYPE
}

\author{
Admi Nazra \\ Department of Mathematics \\ Faculty of Mathematics and Natural Science \\ Universitas Andalas, Indonesia \\ nazra@sci.unand.ac.id
}

\section{Lyra Yulianti}

Department of Mathematics

Faculty of Mathematics and Natural Sciences

Universitas Andalas, Indonesia

lyra@sci.unand.ac.id

\author{
Zulakmal \\ Department of Mathematics \\ Faculty of Mathematics and Natural Sciences \\ Universitas Andalas, Indonesia \\ zulakmal@ sci.unand.ac.id
}

Muhafzan*

Department of Mathematics

Faculty of Mathematics and Natural Sciences

Universitas Andalas, Indonesia

muhafzan@sci.unand.ac.id

Article history:

Received 10.10.2020, Accepted 10.12.2020

\begin{abstract}
In this article, the linear quadratic optimization problem subject to fractional order differential algebraic systems of Riemann-Liouville type is studied. The goal of this article is to find the optimal control-state pairs satisfying the dynamic constraint of the form a fractional order differential algebraic systems such that the linear quadratic objective functional is minimized. The transformation method is used to find the optimal controlstate pairs for this problem. The optimal control-state pairs is stated in terms of Mittag-Leffler function.
\end{abstract}

\section{Key words}

Fractional order, differential algebraic system, Riemann-Liouville fractional derivative, Mittag-Leffler function.

\section{Introduction}

Recently, many issues in the physical field have used the optimal control theory for problem solving. This information can be found in literatures such as [Frank et all, 2016], [Melendez and Santos, 2017], [Arafa et all, 2017], [Anbarasi and Kanthalakshmi, 2016]. As reported in [Frank et all, 2016], the optimal control theory is applied for a complex atomic quantum system.

The linear quadratic optimization for fractional order differential algebraic systems is a specific optimal con- trol problem of the following form:

$$
\begin{gathered}
\min _{\varrho} J(\varrho, \zeta)=\int_{0}^{1}(\langle\zeta, Q \zeta\rangle+\langle\varrho, R \varrho\rangle) d t, \\
\text { s.t. }\left(E \mathcal{D}_{t}^{\alpha}-A\right) \zeta=B \varrho, \zeta(0)=\zeta_{0},
\end{gathered}
$$

where $\langle\cdot, \cdot\rangle$ denotes inner product of two vectors, $\zeta=\zeta(t) \in \mathbb{R}^{n}$ denotes state, $\varrho=\varrho(t) \quad \in$ $\mathbb{R}^{r}$ denotes control, $E, A \in \mathbb{R}^{n \times n}$ with $\operatorname{rank}(E)<n$, $B \in \mathbb{R}^{n \times r}, Q$ and $R$ are symmetric positive definite matrices. In the dynamic constraint (2), $\mathcal{D}_{t}^{\alpha}$ denotes the fractional derivative operator of order $\alpha, \alpha \in$ $(m-1, m)$ with $m \in \mathbb{N}$. The dynamic constraint in equation (2) is called a fractional differential algebraic system [Muhafzan et all, 2019]. It can be proved that the solution of fractional differential algebraic system (2) exists if $\operatorname{det}\left(s^{\alpha} E-A\right) \neq 0$ for some $s \in \mathbb{C}$ [Batiha et all, 2018]. The dynamical system of this kind for the matrix $E=I$ has been discussed in [Khanduzi et all, 2020], [Evirgen, 2016] and [Evirgen, 2017]. Note that for $\alpha=1$, the operator $\mathcal{D}_{t}^{\alpha}$ constitutes an usual derivative and this already studied in [Petrenko et all, 2020], [Zulakmal et all, 2018] and [Muhafzan, 2010]. An application of the optimal control problem (1) and (2) in mechanical descriptor system for $\alpha=1$ is given in [Muller, 1999].

It is well known that the problem to be solved in the optimization problem (1) and (2) is to find the control- 
state pairs $(\varrho, \zeta)$ satisfying the fractional dynamic constraint (2) such that the objective functional (1) is minimized. To the best of the author's knowledge, little work has been done with the optimization problem (1) subject to the fractional dynamic system (2). However, this issue was discussed in [Chiranjeevi and Biswas, 2020] recently, for which $\mathcal{D}_{t}^{\alpha}$ is the fractional derivative operator in terms of the Caputo.

In this paper we discuss the linear quadratic optimization problem of infinite horizon subject to differential algebraic system of fractional order of the following form:

$$
\begin{gathered}
\min _{\varrho} \mathcal{J}(\varrho, \zeta)=\int_{0}^{\infty}(\langle\zeta, Q \zeta\rangle+\langle\varrho, R \varrho\rangle) d t, \\
\text { s.t. } \quad\left(E \mathcal{D}_{t}^{\alpha}-A\right) \zeta=B \varrho, \zeta(0)=\zeta_{0},
\end{gathered}
$$

where $\mathcal{D}_{t}^{\alpha}$ is the fractional derivative in terms of

Riemann-Liouville of order $\alpha \in(0,1)$. The aim of this paper is to find the control-state pairs $(\varrho, \zeta)$ satisfying the fractional dynamic constraint (4) such that the objective functional (3) is minimized. The solving method is to transform the linear quadratic optimization (3) and (4) into the standard fractional linear quadratic optimization. Using the theory for the standard fractional linear quadratic optimization, we find the optimal control-state pairs $(\varrho, \zeta)$ for the optimization problem (3) and (4) for which they are stated in a combination of the MittagLeffler functions. Indeed the linear quadratic optimization problem (3) and (4) constitutes an extension of the linear quadratic optimization problem proposed in [Chiranjeevi and Biswas, 2020]. Therefore the results of this paper constitute a new contribution in the field of optimization subject for fractional differential algebraic dynamic system. Moreover, this result can be also used to extent the results in [Muller, 1999] on the linear mechanical descriptor systems for fractional derivative of order $\alpha$, with $\alpha \in(0,1)$.

The rest of the paper is organized as follows. Section 2 considers some preliminaries information about the Riemann-Liouville fractional derivative, Mittag-Leffler function and fractional order differential equation system. Section 3 presents the transformation process the linear quadratic optimization problem subject to fractional order differential algebraic system into the standard fractional linear quadratic optimization problem. The main result of this article and a numerical example illustrating the results is also given in section 3. Section 4 concludes the paper.

\section{Preliminaries Information}

There are several mathematical tools used in this study. The following statement is the definition of the fractional order Riemann-Liouville derivative and Mittag-Leffler function. Let $\zeta:[0, \infty) \rightarrow \mathbb{R}^{n}$ be an integrable function. The Riemann-Liouville fractional derivative of order $\alpha$ with $\alpha \in(m-1, m), m \in \mathbb{N}$, is defined by

$$
\mathcal{D}_{t}^{\alpha} \zeta(t)=\frac{1}{\Gamma(m-\alpha)} \frac{d^{m}}{d t^{m}} \int_{0}^{t}(t-\tau)^{m-\alpha-1} \zeta(\tau) d \tau,
$$

where $\Gamma($.$) is the Euler Gamma function [Batiha et all,$ 2018]. One can easily find that the Riemann-Liouville fractional derivative of order $\alpha \in(m-1, m), m \in \mathbb{N}$ for a constant function $c$ is $\frac{c t^{-\alpha}}{\Gamma(m-\alpha)}$. It is clear that such a derivative is different from the usual derivative of a constant function $c$.

The one parameter Mittag-Leffler function is defined by

$$
\mathcal{E}_{\beta}(z)=\sum_{j=0}^{\infty} \frac{z^{j}}{\Gamma(j \beta+1)}, z \in \mathbb{C},
$$

where $\beta>0$ [Batiha et all, 2018], [Evirgen and Ozdemir, 2011]. One can replace variable $z$ in (6) by $A z$ for an arbitrary square matrix $A$, such that

$$
\mathcal{E}_{\beta}(A z)=\sum_{j=0}^{\infty} \frac{A^{j} z^{j}}{\Gamma(j \beta+1)} .
$$

It is easy to see that

$$
\mathcal{E}_{1}(A z)=\sum_{j=0}^{\infty} \frac{A^{j} z^{t}}{\Gamma(j+1)}=\sum_{j=0}^{\infty} \frac{A^{j} z^{j}}{j !}=\exp (A z) .
$$

The two parameters Mittag-Leffler function for $A z$ was given by:

$$
\mathcal{E}_{\beta, \gamma}(A z)=\sum_{j=0}^{\infty} \frac{A^{j} z^{j}}{\Gamma(j \beta+\gamma)},
$$

where $\beta, \gamma>0$. It is clear that $\mathcal{E}_{\beta, 1}(A z)=\mathcal{E}_{\beta}(A z)$. The Mittag-Leffler function (6) and (9) are convergent series [Batiha et all, 2018].

The Mittag-Leffler play an important role in solving the system of the following fractional differential equations:

$$
\mathcal{D}_{t}^{\alpha} \zeta=A \zeta+\varrho, \zeta(0)=\zeta_{0}, 0<\alpha<1,
$$

where $\mathcal{D}_{t}^{\alpha}$ is the Riemann-Liouville fractional derivative. Using the Laplace transformation one can easily prove the following theorem.

Theorem 1. [Hristova et all, 2020] The solution of system (10) is

$$
\zeta(t)=t^{\alpha-1} \mathcal{E}_{\alpha}\left(A t^{\alpha}\right) \zeta_{0}+\int_{0}^{t}(t-s)^{\alpha-1} \mathcal{E}_{\alpha, \alpha}\left(A t^{\alpha}\right) \varrho(s) d s,
$$

for $t \in[0, T]$. 


\section{Transformation and Solution}

Reconsider the linear quadratic optimization problem (3) and (4). A control-state pair $(\varrho, \zeta)$ is called admissible for the optimization problem (3) and (4) if it satisfies the constraint (4) for an initial state $\zeta_{0} \in \mathbb{R}^{n}$ and $\mathcal{J}(\varrho, \zeta)<\infty$. A control-state pair $\left(\varrho^{*}, \zeta^{*}\right)$ is called an optimal control-state pair for the optimization problem (3) and (4) if it is an admissible and $\mathcal{J}\left(\varrho^{*}, \zeta^{*}\right)=$ $\min \mathcal{J}(\varrho, \zeta)$. Let us define the admissible control-state pairs set for the optimization problem (3) and (4) by

$$
\begin{aligned}
\mathcal{Y} \triangleq & \{(\varrho, \zeta) \mid(\varrho, \zeta) \text { is continuous satisfies }(4) \\
& \text { and } \mathcal{J}(\varrho, \zeta)<\infty\} .
\end{aligned}
$$

The problem under consideration is how the explicit formulation of the optimal control-state pairs $\left(\varrho^{*}, \zeta^{*}\right) \in$ $\mathcal{Y}$ such that

$$
\mathcal{J}\left(\varrho^{*}, \zeta^{*}\right)=\min _{\varrho} \mathcal{J}(\varrho, \zeta) .
$$

First of all, let us transform the linear quadratic optimization problem (3) and (4) into the standard fractional linear quadratic optimization problem. For this purpose, we adopt the Definition 1 in [Fang et all, 2014] and the Singular Value Decomposition(SVD) Theorem [Klema and Laub, 1980] to find a restricted system equivalent (r.s.e.) to the system (4).

Definition 1. A fractional order differential algebraic system

$$
\left(\bar{E} \mathcal{D}_{t}^{\alpha}-\bar{A}\right) \breve{\zeta}=\bar{B} \varrho, \breve{\zeta}(0)=\breve{\zeta}_{0}
$$

is said to be a restricted system equivalent (r.s.e.) to the system (4) if there exist two nonsingular matrices $U, V \in$ $\mathbb{R}^{n \times n}$ such that $U E V=\bar{E}, U A V=\bar{A}, U B=\bar{B}$ and $\zeta=V \breve{\zeta}$.

Obviously, the restricted system equivalence is an equivalent relationship and it is consistent with Definition 1 in [Fang et all, 2014] for the standard differential algebraic systems.

Let $\operatorname{rank}(E)=p<n$. Base on the Singular Value Decomposition(SVD) Theorem [Klema and Laub, 1980], there exist the nonsingular matrices $U, V \in \mathbb{R}^{n \times n}$ such that

$$
U E V=\operatorname{diag}\left(I_{p}, O\right),
$$

where $I_{p}$ is an identity matrix of size $p \times p$ and $O$ is a zero matrix. Using these $U$ and $V$ matrices, we have the following fractional order differential algebraic system

$$
\left(\operatorname{diag}\left(I_{p}, O\right) \mathcal{D}_{t}^{\alpha}-\left[\begin{array}{ll}
A_{11} & A_{12} \\
A_{21} & A_{22}
\end{array}\right]\right)\left[\begin{array}{l}
\zeta_{1} \\
\zeta_{2}
\end{array}\right]=\left[\begin{array}{l}
B_{1} \\
B_{2}
\end{array}\right] \varrho,
$$

with $\zeta_{1}(0)=\zeta_{10}$, which is r.s.e. to the fractional dynamic constraint (4), where

$$
\left[\begin{array}{ll}
A_{11} & A_{12} \\
A_{21} & A_{22}
\end{array}\right]=U A V,\left[\begin{array}{l}
B_{1} \\
B_{2}
\end{array}\right]=U B,\left[\begin{array}{l}
\zeta_{1} \\
\zeta_{2}
\end{array}\right]=V^{-1} \zeta
$$

with $A_{11} \in \mathbb{R}^{p \times p}, B_{1} \in \mathbb{R}^{p \times r}, \zeta_{1} \in \mathbb{R}^{p}$ and $\zeta_{10}=$ $\left[I_{p} O\right] U \zeta_{0}$. Assume that the system (4) is impulse controllable. One can observe that the transformation (13) and (15) implies the system (14) is also impulse controllable, see [Zulakmal et all, 2018]. This is equivalent to

$$
\operatorname{rank}\left[\begin{array}{ll}
A_{22} & B_{2}
\end{array}\right]=n-p \text {. }
$$

Using the transformation $V^{-1} \zeta=\left[\begin{array}{l}\zeta_{1} \\ \zeta_{2}\end{array}\right]$, the objective function (3) can be replaced with

$$
\begin{aligned}
\min _{\varrho} \mathcal{J}(\varrho, \zeta)= & \int_{0}^{\infty}\left(\left\langle\zeta_{1}, Q_{11} \zeta_{1}\right\rangle+2\left\langle\zeta_{1}, Q_{12} \zeta_{2}\right\rangle\right. \\
& \left.+\left\langle\zeta_{2}, Q_{22} \zeta_{2}\right\rangle+\langle\varrho, R \varrho\rangle\right) d t,
\end{aligned}
$$

where $\left[\begin{array}{ll}Q_{11} & Q_{12} \\ Q_{12}^{\top} & Q_{22}\end{array}\right]=V^{\top} Q V$ with $Q_{11} \in \mathbb{R}^{p \times p}, Q_{12} \in$ $\mathbb{R}^{p \times(n-p)}$ and $Q_{22} \in \mathbb{R}^{(n-p) \times(n-p)}$. Using the condition (16), the solution of equation (14) is

$$
\left[\begin{array}{c}
\zeta_{2} \\
\varrho
\end{array}\right]=\left[\begin{array}{ll}
-\hat{A}^{\dagger} A_{21} \Phi
\end{array}\right]\left[\begin{array}{l}
\zeta_{1} \\
\mathbf{v}
\end{array}\right]
$$

for some full rank matrix $\Phi \in \mathbb{R}^{(n-p+r) \times r}$ with $\Phi \in \operatorname{ker}\left[\begin{array}{ll}A_{22} & B_{2}\end{array}\right], \quad \mathbf{v} \in \mathbb{R}^{r}$ and $\hat{A}^{\dagger}=$ $\left[\begin{array}{ll}A_{22} & B_{2}\end{array}\right]^{\top}\left(\left[\begin{array}{ll}A_{22} & B_{2}\end{array}\right]\left[\begin{array}{l}A_{22}^{\top} \\ B_{2}^{\top}\end{array}\right]\right)^{-1}$ is the generalized inverse of the matrix $\left[A_{22} B_{2}\right]$. Using the expression (18), the following transformation is created:

$$
\left[\begin{array}{c}
\zeta_{1} \\
-- \\
\zeta_{2} \\
\varrho
\end{array}\right]=\left[\begin{array}{cc}
I_{p} & O \\
-\hat{A}^{\dagger} A_{21} & \Phi
\end{array}\right]\left[\begin{array}{c}
\zeta_{1} \\
\mathbf{v}
\end{array}\right] .
$$

By substituting (19) into (17), we obtain the following linear quadratic optimization problem:

$$
\begin{gathered}
\min _{\mathbf{v}} \mathcal{J}\left(\mathbf{v}, \zeta_{1}\right)=\int_{0}^{\infty}\left(\left\langle\zeta_{1}, \bar{Q}_{11} \zeta_{1}\right\rangle+2\left\langle\zeta_{1}, \bar{Q}_{12} \mathbf{v}\right\rangle\right. \\
\left.+\left\langle\mathbf{v}^{\top}, \bar{Q}_{22} \mathbf{v}\right\rangle\right) d t \\
\text { s.t. }\left(\mathcal{D}_{t}^{\alpha}-\bar{A}\right) \zeta_{1}=\bar{B} \mathbf{v}, \zeta_{1}(0)=\zeta_{10}
\end{gathered}
$$

where

$$
\bar{A}=A_{11}-\left[\begin{array}{ll}
A_{11} & B_{1}
\end{array}\right] \hat{A}^{\dagger} A_{21}, \bar{B}=\left[\begin{array}{ll}
A_{11} & B_{1}
\end{array}\right] \Phi,
$$




$$
\begin{aligned}
& \bar{Q}_{11}=Q_{11}+\left(\hat{A}^{\dagger} A_{21}\right)^{\top}\left[\begin{array}{cc}
Q_{22} & O \\
O & R
\end{array}\right] \hat{A}^{\dagger} A_{21}, \\
& \bar{Q}_{12}=\left[Q_{12} O\right] \Phi-\left(\hat{A}^{\dagger} A_{21}\right)^{\top}\left[\begin{array}{cc}
Q_{22} & O \\
O & R
\end{array}\right] \Phi, \\
& \bar{Q}_{21}=\Phi^{\top}\left[\begin{array}{c}
Q_{21} \\
O
\end{array}\right]-\Phi^{\top}\left[\begin{array}{cc}
Q_{22} & O \\
O & R
\end{array}\right] \hat{A}^{\dagger} A_{21}, \\
& \bar{Q}_{22}=\Phi^{\top}\left[\begin{array}{cc}
Q_{22} & O \\
O & R
\end{array}\right] \Phi .
\end{aligned}
$$

One can see that the linear quadratic optimization problem (3) and (4) is equivalent to the standard linear quadratic optimization problem (20) with the state $\zeta_{1}$ and the control v. Furthermore, the optimal control-state pairs $\left(\varrho^{*}, \zeta^{*}\right)$ can be found by solving the standard fractional linear quadratic optimization problem (20).

One can observe that the positive definite assumption of the matrix $Q$ and $R$ implies $\bar{Q}_{22}$ in equation (21) is also positive definite. Therefore, one can use the theory in [Matychyn and Onyshchenko, 2018] and [Li and Chen, 2008] regarding the standard fractional linear quadratic optimization problem. Using the results in [Matychyn and Onyshchenko, 2018], the optimal state pairs $\left(\mathbf{v}^{*}, \zeta_{1}^{*}\right)$ for optimization problem (20) exists and unique if

$$
\operatorname{rank}\left(\left[\bar{B}|\bar{A} \bar{B}| \ldots \mid \bar{A}^{p-1} \bar{B}\right]\right)=p .
$$

The control that minimizes $\mathcal{J}\left(\mathbf{v}, \zeta_{1}\right)$ is given by

$$
\mathbf{v}^{*}=-\bar{Q}_{22}^{-1}\left(\bar{Q}_{12}^{\top}+\bar{B}^{\top} S\right) \zeta_{1}^{*},
$$

where the state $\zeta_{1}^{*}$ is the solution of the following fractional differential equation:

$$
\left(\mathcal{D}_{t}^{\alpha}-\mathcal{K}\right) \zeta_{1}=\mathbf{0}, \zeta_{1}(0)=\zeta_{10},
$$

where $\mathcal{K}=\bar{A}-\bar{B} \bar{Q}_{22}^{-1}\left(\bar{Q}_{12}^{\top}+\bar{B}^{\top} S\right)$ with $S$ is the unique positive definite solution of the following algebraic Riccati equation:

$\bar{A}^{\top} S+S \bar{A}+\bar{Q}_{11}-\left(S \bar{B}+\bar{Q}_{12}\right) \bar{Q}_{22}^{-1}\left(S \bar{B}+\bar{Q}_{12}\right)^{\top}=O$.

Using the equation (11), the solution of equation (23) is given by

$$
\zeta_{1}(t)=t^{\alpha-1} \mathcal{E}_{\alpha}\left(\mathcal{K} t^{\alpha}\right) \zeta_{10} .
$$

Likewise, using the transformation (15) and (19), the optimal control-state pairs $\left(\varrho^{*}, \zeta^{*}\right)$ of the linear quadratic optimization problem (3) and (4) is given by

$$
\begin{aligned}
{\left[\begin{array}{l}
\zeta \\
\varrho
\end{array}\right] } & =\left[\begin{array}{ll}
N & O \\
O & I_{r}
\end{array}\right]\left[\begin{array}{c}
\zeta_{1} \\
\zeta_{2} \\
\varrho
\end{array}\right] \\
& =\left[\begin{array}{ll}
N & O \\
O & I_{r}
\end{array}\right]\left[\begin{array}{cc}
I_{p} & O \\
\mathcal{A}_{1} & \Phi_{1} \\
\mathcal{A}_{2} & \Phi_{2}
\end{array}\right]\left[\begin{array}{c}
I_{p} \\
-Q_{22}^{-1}\left(Q_{12}^{\top}+B^{\top} S\right)
\end{array}\right] \zeta_{1} \\
& =\left[\begin{array}{ll}
N & O \\
O & I_{r}
\end{array}\right]\left[\begin{array}{c}
I_{p} \\
\mathcal{A}_{1}-\Phi_{1} Q_{22}^{-1}\left(Q_{12}^{\top}+B^{\top} S\right) \\
\mathcal{A}_{2}-\Phi_{2} Q_{22}^{-1}\left(Q_{12}^{\top}+B^{\top} S\right)
\end{array}\right] \zeta_{1},
\end{aligned}
$$

or in a separate form given by

$$
\varrho^{*}=t^{\alpha-1}\left(\mathcal{A}_{2}-\Phi_{2} \bar{Q}_{22}^{-1}\left(\bar{Q}_{12}^{\top}+\bar{B}^{\top} S\right)\right) \mathcal{E}_{\alpha}\left(\mathcal{K} t^{\alpha}\right) \zeta_{10}
$$

and

$\zeta^{*}=t^{\alpha-1} N\left[\begin{array}{c}I_{p} \\ \mathcal{A}_{1}-\Phi_{1} \bar{Q}_{22}^{-1}\left(\bar{Q}_{12}^{\top}+\bar{B}^{\top} S\right)\end{array}\right] \mathcal{E}_{\alpha}\left(\mathcal{K} t^{\alpha}\right) \zeta_{10}$,

where $\left[\begin{array}{l}\mathcal{A}_{1} \\ \mathcal{A}_{2}\end{array}\right]=-\hat{A}^{\dagger} A_{21}, \quad \Phi=\left[\begin{array}{l}\Phi_{1} \\ \Phi_{2}\end{array}\right], \quad \begin{array}{lll}\mathcal{A}_{1} & \in\end{array}$ $\mathbb{R}^{(n-p) \times p}, \mathcal{A}_{2} \in \mathbb{R}^{r \times p}, \Phi_{1} \in \mathbb{R}^{(n-p) \times r} \quad$ and $\Phi_{2} \in \mathbb{R}^{r \times r}$.

One can see that the optimal control-state pairs is stated in terms of Mittag-Leffler function.

In order to illustrate the results, let us consider the linear quadratic optimization problem (3) and (4) where the matrices $E, A, B, Q$ and $R$ are given as follows:

$$
\begin{aligned}
& E=\left[\begin{array}{cccc}
2 & 0 & 0 & 0 \\
0 & 1 & -1 & 0 \\
0 & 0 & 0 & 0 \\
0 & 0 & 0 & 0
\end{array}\right], A=\left[\begin{array}{rrrr}
-1 & 0 & 1 & 0 \\
-1 & 0 & -1 & 0 \\
0 & -2 & 2 & -1 \\
1 & 2 & -1 & 0
\end{array}\right], \\
& B=\left[\begin{array}{l}
6 \\
0 \\
3 \\
1
\end{array}\right], Q=\left[\begin{array}{cccc}
2 & 1 & -7 & -4 \\
1 & 1 & -4 & -3 \\
-7 & -4 & 25 & 15 \\
-4 & -3 & 15 & 10
\end{array}\right]
\end{aligned}
$$

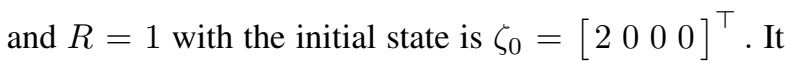
is clear that $p=2$. By taking the matrices

$U=\left[\begin{array}{cccc}0.5 & 0 & 0 & 0 \\ 0 & 0.7071 & 0 & 0 \\ 0 & 0 & 0 & 1 \\ 0 & 0 & 1 & 0\end{array}\right], V=\left[\begin{array}{cccc}1 & 0 & 0 & 0 \\ 0 & 0.7071 & 0 & 0.7071 \\ 0 & -0.7071 & 0 & 0.7071 \\ 0 & 0 & 1 & 1\end{array}\right]$

we have

$$
U E V=\left[\begin{array}{ll}
I_{2} & O \\
O & O
\end{array}\right]
$$

It is easy to verify that $\operatorname{rank}\left[A_{22} B_{2}\right]=2$, thus the fractional differential algebraic system (14) is impulse controllable. By choosing

$$
\Phi=\left[\begin{array}{lll}
-0.4082 & 0.8660 & 0.2887
\end{array}\right]^{\top} \in \operatorname{ker}\left[\begin{array}{ll}
A_{22} & B_{2}
\end{array}\right],
$$

the problem (3) and (4) can be equivalently changed into the standard fractional linear quadratic optimization problem (20) where $\zeta_{1} \in \mathbb{R}^{2}, \mathbf{v} \in \mathbb{R}$ with

$$
\bar{A}=\left[\begin{array}{cc}
-1.4167 & -0.5303 \\
-0.1179 & 2.2500
\end{array}\right], \bar{B}=\left[\begin{array}{l}
0.7217 \\
0.2041
\end{array}\right],
$$




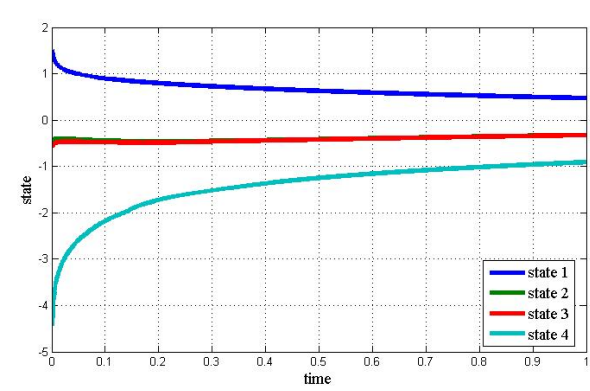

Figure 1. State trajectories for $\alpha=0.9$

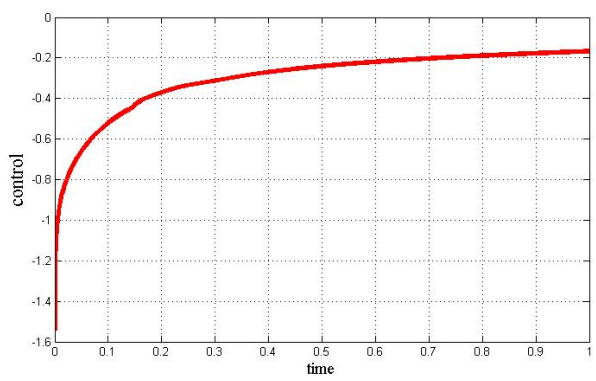

Figure 2. Control trajectory for $\alpha=0.9$

$$
\begin{gathered}
\zeta_{1}(0)=\left[\begin{array}{l}
1 \\
0
\end{array}\right], \bar{Q}_{11}=\left[\begin{array}{cc}
41 & 126.5721 \\
126.5721 & 392.500
\end{array}\right], \\
\bar{Q}_{12}=\left[\begin{array}{c}
-8.6603 \\
-28.1691
\end{array}\right], \bar{Q}_{22}=3 .
\end{gathered}
$$

Since $\operatorname{rank}([\bar{B} \mid \bar{A} \bar{B}])=p=2$, the control that minimizes $\mathcal{J}\left(\mathbf{v}, \zeta_{1}\right)$ is given by

$$
\mathbf{v}^{*}=-[0.439732 .7423] \zeta_{1}^{*},
$$

where the state $\zeta_{1}^{*}$ is the solution of the following fractional differential equation:

$$
\left(\mathcal{D}_{t}^{\alpha}-\left[\begin{array}{ll}
-1.7340 & -24.1601 \\
-0.2076 & -4.4335
\end{array}\right]\right) \zeta_{1}=\left[\begin{array}{l}
0 \\
0
\end{array}\right],
$$

with $\zeta_{1}(0)=\left[\begin{array}{l}1 \\ 0\end{array}\right]$, and the solution of the algebraic Riccati equation (24) is given by the matrix

$$
S=\left[\begin{array}{cc}
14.4483 & -2.1935 \\
-2.1935 & 626.9672
\end{array}\right] \text {. }
$$

The solution of the fractional differential equation (27) is

$$
\begin{aligned}
\zeta_{1}(t) & =t^{\alpha-1} \mathcal{E}_{\alpha}\left(\left[\begin{array}{l}
-1.7340-24.1601 \\
-0.2076
\end{array}\right] t^{\alpha}\right)\left[\begin{array}{l}
1 \\
0
\end{array}\right] \\
& =t^{\alpha-1} \sum_{j=0}^{\infty}\left[\begin{array}{l}
0.7581(-0.47)^{j} \frac{t^{j \alpha}}{\Gamma(j \alpha+1)} \\
0.0397(-5.70)^{j} \frac{t^{j \alpha}}{\Gamma(j \alpha+1)}
\end{array}\right] \\
& =t^{\alpha-1}\left[\begin{array}{l}
0.7581 \mathcal{E}_{\alpha}\left(-0.47 t^{\alpha}\right) \\
0.0397 \mathcal{E}_{\alpha}\left(-5.70 t^{\alpha}\right)
\end{array}\right],
\end{aligned}
$$

thus

$$
\begin{aligned}
\mathbf{v}^{*} & =-t^{\alpha-1}\left[\begin{array}{c}
0.4397 \\
32.7423
\end{array}\right]^{\top}\left[\begin{array}{c}
0.7581 \mathcal{E}_{\alpha}\left(-0.47 t^{\alpha}\right) \\
0.0397 \mathcal{E}_{\alpha}\left(-5.70 t^{\alpha}\right)
\end{array}\right] \\
& =-t^{\alpha-1}\left(0.33 \mathcal{E}_{\alpha}\left(-0.47 t^{\alpha}\right)+1.3 \mathcal{E}_{\alpha}\left(-5.70 t^{\alpha}\right)\right) .
\end{aligned}
$$

Using (25) and (26) we find

$$
\zeta^{*}=t^{\alpha-1}\left[\begin{array}{c}
0.76 \mathcal{E}_{\alpha}\left(-0.47 t^{\alpha}\right) \\
-0.54 \mathcal{E}_{\alpha}\left(-0.47 t^{\alpha}\right)+0.31 \mathcal{E}_{\alpha}\left(-5.70 t^{\alpha}\right) \\
-0.54 \mathcal{E}_{\alpha}\left(-0.47 t^{\alpha}\right)+0.25 \mathcal{E}_{\alpha}\left(-5.70 t^{\alpha}\right) \\
-1.43 \mathcal{E}_{\alpha}\left(-0.47 t^{\alpha}\right)-0.80 \mathcal{E}_{\alpha}\left(-5.70 t^{\alpha}\right)
\end{array}\right]
$$

and

$\varrho^{*}=-t^{\alpha-1}\left(0.25 \mathcal{E}_{\alpha}\left(-0.47 t^{\alpha}\right)+0.3613 \mathcal{E}_{\alpha}\left(-5.70 t^{\alpha}\right)\right)$.

The state trajectories $\zeta^{*}$ for $\alpha=0.9$ is shown in Figure 1 and the control trajectory $\varrho^{*}$ is shown in Figure 2.

\section{Conclusion}

We have found the explicit formulation of optimal control-state pairs for the linear quadratic optimization problem subject to fractional order differential algebraic system of Riemann-Liouville type. The optimal controlstate pairs is stated in terms of Mittag-Leffler function. An example illustrating the optimal control-state pairs has been presented.

Acknowledgment. This work was supported by Universitas Andalas under Grant KRP1GB-PDU-Unand-2019 No. T/8/UN.16.17/PP.IS-KRP1GB/LPPM/2019.

\section{References}

Arafa, N., Gama, S. and Pereira, F. L. (2017) Optimization and control theory in shell models of turbulence. In Physcon, Florence, Italy, July 17-19.

Anbarasi, M. P. and Kanthalakshmi, S. (2016) Linear quadratic optimal control of solar photovoltaic system: An experimental validation. Journal of Renewable and Sustainable Energy, 8 (053502).

Batiha, I. M., El-Khazali, R., Alsaedi, A. and Shaher, M. (2018) The general solution of singular fractionalorder linear time-invariant continuous systems with regular pencils. Entropy, 20(6), pp. 1-13.

Chiranjeevi, T and Biswas, R. K. (2020) Linear Quadratic Optimal Control Problem of Fractional Order Continuous-Time Singular System. In Procedia Computer Science, 171, pp. 1261-1268. 
Evirgen, F. and Ozdemir, N. (2011) Multistage Adomian decomposition method for solving NLP problems over a nonlinear fractional dynamical system. Journal of Computational and Nonlinear Dynamics, 6(2), pp. 16.

Evirgen, F. (2017) Conformable fractional gradientbased dynamic system for constrained optimization problem. Acta Physica Polonica A, 132(3), pp. 10661069.

Evirgen, F. (2016) Analyze the optimal solutions of optimization problems by means of fractional gradientbased system using VIM. An International Journal of Optimization and Control: Theories \& Applications, 6(2), pp. 75-83.

Fang, Q., Zhang, B. and Feng, J. (2014) Singular LQ problem for irregular singular systems. Journal of Applied Mathematics, 853415, pp. 1-9.

Frank, S. V., Bonneau, M., Schmiedmayer, J., Hild, S., Gross, C., Cheneau. M., Bloch, I., Pichler, T., Negretti, A., Calarco, T. and Montangero, S. (2016) Optimal control of complex atomic quantum systems. Scientific Reports, 6, pp. 1-12.

Hristova, S., Agarwal, R. and O'Regan. D. (2020) Explicit solutions of initial value problems for systems of linear Riemann-Liouville fractional differential equations with constant delay. Advances in Difference Equations, 180, pp. 1-18.

Khanduzi, R., Ebrahim, Z. and Beik, S. P. A. (2020) Optimal control of fractional integro-differential systems based on a spectral method and grey wolf optimizer. An International Journal of Optimization and Control: Theories \& Applications, 10(1), pp. 55-65.

Klema, V.C. and Laub, A. J. (1980) The singular value decomposition: its computation and some applications. IEEE Transactions on Automatic Control, 25(2), pp. 164-176.

Li, Y. and Chen, Y. Q. (2008) Fractional order linear quadratic regulator. In Proceeding of IEEE/ASME International Conference on Mechtronic and Embedded Systems and Applications, Beijing, China, Oct. 12-15, pp. 363-368.

Matychyn, I. and Onyshchenko, V. (2018) Optimal control of linear systems with fractional derivatives. Fractional Calculus \& Applied Analysis, 1, pp. 134150.

Melendez, C. R. and Santos, L. G. (2017) Numerical approximation in optimal control of two-level quantum systems. In Physcon, Florence, Italy, July 17-19.

Muhafzan, Nazra, A., Yulianti, L. and Zulakmal (2019) On positive normalization of fractional order continuous disturbance differential algebraic system. Cybernetics and Physics, 8(1), pp. 34-38.

Muhafzan (2010) Use of semidefinite programming for solving the LQR problem subject to descriptor systems. Int. J. Math. Computh. Sci., 20(4), pp. 655-664.

Muller, P. C. (1999) Linear-Quadratic Optimal Control of Descriptor Systems. Journal of the Brazilian Society of Mechanical Sciences, 21(3), pp. 423-432.

Petrenko, P., Samsonyuk, O. and Staritsyn, M. (2020) A note on differential-algebraic systems with impulsive and hysteresis phenomena. Cybernetics and Physics, 9(1), pp. 51-56.

Zulakmal, Narwen, Rudianto, B., Baqi, A. I. and Muhafzan (2018) On the LQ optimization subject to descriptor system under disturbance. Asian Journal of Scientific Research, 11(4), pp. 540-543. 